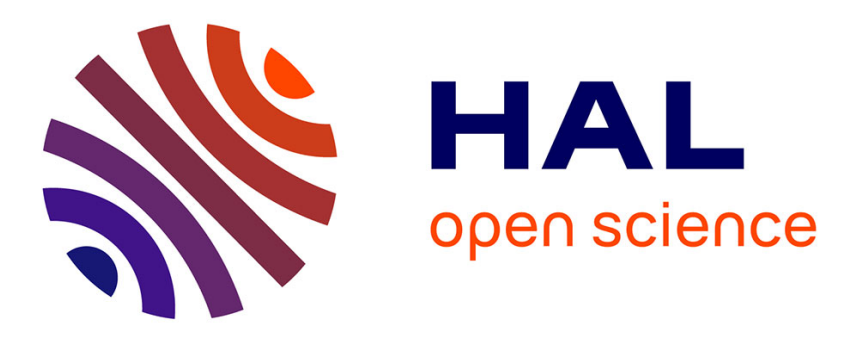

\title{
Time integration algorithm based on divergent series resummation, for ordinary and partial differential equations
}

\author{
Dina Razafindralandy, Aziz Hamdouni
}

\section{- To cite this version:}

Dina Razafindralandy, Aziz Hamdouni. Time integration algorithm based on divergent series resummation, for ordinary and partial differential equations. Journal of Computational Physics, 2013, 236, pp.56-73. 10.1016/j.jcp.2012.10.022 . hal-02086543

HAL Id: hal-02086543

https://hal.science/hal-02086543

Submitted on 1 Apr 2019

HAL is a multi-disciplinary open access archive for the deposit and dissemination of scientific research documents, whether they are published or not. The documents may come from teaching and research institutions in France or abroad, or from public or private research centers.
L'archive ouverte pluridisciplinaire HAL, est destinée au dépôt et à la diffusion de documents scientifiques de niveau recherche, publiés ou non, émanant des établissements d'enseignement et de recherche français ou étrangers, des laboratoires publics ou privés. 


\title{
Time integration algorithm based on divergent series resummation, for ordinary and partial differential equations
}

\author{
Dina Razafindralandy*, Aziz Hamdouni \\ Laboratoire des Sciences de l'Ingénieur pour l'Environnement (LaSIE) \\ Université de La Rochelle, France
}

\begin{abstract}
Borel's technique of divergent series resummation is transformed into a numerical code and used as a time integration scheme. It is applied to the resolution of regular and singular problems arising in fluid mechanics. Its efficiency is compared to those of classical discretization schemes.
\end{abstract}

Keywords: Peturbation method, divergent series, Borel resummation, Fluid Mechanics

\section{Introduction}

The resolution of many evolution problems in fluid mechanics, such as an unsteady flow, requires a long computation time because the time step allowed by the numerical scheme is generally very small. For example, the Euler explicit method can be applied to the resolution of a diffusion problem only under a very restrictive stability condition on $\Delta t$. This time step can be increased with an implicit method, but with the cost of a matrix inversion at each time step. Moreover, many standard discretization schemes give only snapshots of the solution at each time step but not its continuous evolution. These shemes may then miss fast variations or fluctuations of the solution. An alternative to these schemes is the decomposition of the solution into a

\footnotetext{
${ }^{*}$ Corresponding author. LaSIE, av. Michel Crépeau. 17042 La Rochelle Cedex, France. Email addresses: drazafin@univ-lr.fr (Dina Razafindralandy), ahamdoun@univ-lr.fr (Aziz Hamdouni)
} 
time series. When the non-linearity of the problem is algebraic (it is the case of the Navier-Stokes equations), this procedure transforms the initial problem into a cascade of linear and explicit problems. The approximate solution is then valid, not at discrete time steps, but in a whole interval, corresponding to the (numerical) radius of convergence of the series. To reach high values of $t$, a continuation procedure is applied, that is, the problem is solved again with the last valid solution as initial condition. The solution is then computed branch-wise. In the whole process, at most one matrix has to be inverted. One other important advantage of the series decomposition is that it gives the asymptotic behaviour of the solution when a blow-up in finite time occurs. These blow-up may arise with the Navier-Stokes equations.

The series decomposition method, called also perturbation or asymptotic method, is particularly efficient in the detection of bifurcations and the computation of bifurcation branches $[8,51,47]$. Numerically, it gave rise to the Asymptotic Numerical Method (ANM) [17] which has been used succesfully in solid mechanics $[20,21,12,11]$. In fluid mechanics, the series decomposition method has been used for the detection of steady bifurcations [14, 58, 15], Coanda effect [3], automatic branch switching [31] and convection problems [44]; the decomposition parameter is, for instance, the Reynolds number.

For the detection of unsteady bifurcations, the decomposition parameter has to be the time. However, a time series decomposition may lead to very small radius of convergence. For example, with a well-behaved initial condition, the radius of convergence of a time-series solution of the discretized Navier-Stoke equations decreases quadratically with the grid size (see appendix). In the continuous case, the radius of convergence may even be zero. This behaviour can more easier be understood with the heat equation:

$$
\frac{\partial u}{\partial t}=\frac{\partial^{2} u}{\partial x^{2}}
$$

associated to the initial condition

$$
u_{0}(x)=\frac{1}{1-x}
$$

The formal power series solution is

$$
u(t, x)=\sum_{k \geq 0} \frac{(2 k) !}{k !} \frac{1}{(1-x)^{2 k+1}} t^{k} .
$$


Series (3) diverges factorially for all $x$ [36] even if the initial condition is analytical. In order to obtain an actual solution, the series has then to be "resummed".

The most promising tool to resum divergent series is the Borel-Laplace resummation method $[9,10]$. This technique is a theoretical procedure which associates to the given (eventually divergent) series an (sectorial) analytic function having the series as asymptotics. And if the series has a non-zero convergence radius, the resummation can be understood as a prolongation procedure outside the convergence disc (see section 3.1). The method is based on the usual Laplace transformation and its inverse, the Borel transformation. Its limit is that the series must be a Gevrey series, i.e. must not "diverge faster than a factorial series". Fortunately, as proved by Maillet [39], formal series which are solution of an algebraic ODE are a Gevrey series. This result has been extended by Malgrange [41] to analytic non-linear ODE's. For PDE's, the theory is not so complete. In fluid mechanics, some equations, such as the heat equation (and some generalization) and the Burgers'equation has been investigated [38, 37, 46, 6, 7]. For the Navier-Stokes equations, Costin et al. recently gave summability conditions to the formal power series [18].

Introduced in the late 19th century, Borel's resummation technique has regained popularity these last three decades. The works of Ecalle on resurgent functions and on accelero-summation [26, 27, 16], and those of Ramis and Malgrange on multi-summability [42, 43] opened many extension perspectives to the initial theory and the way to more and more applications in mathematics and theoretical physics.

Since all the transformations involved in the asymptotic method and in the resummation process are formal, Tounier, Ramis and their collaborators $[57,24]$ built a solver of regular and irregular ODE's, called DESIR, which integrates the resummation technique. Although giving exact solutions, this solver may require too much computer ressource in engineering science, where problems are generally of very high order and requires many time steps. At best, they should be combined to a numeric code. Based on the concept of Newton polygon [40, 49], DESIR deals moreover essentially with linear equations (despite some attempts towards the non-linear case [25, 54]).

This article aims to propose a time integration scheme for the resolution of multi-dimensional evolution problems in fluid mechanics, based on the perturbation method (ANM), and associated to the Borel series resummation method to widen the range of validity of the solution, making thus the algo- 
rithm much faster. This scheme gives as output an analytic function in time. Inheriting from the properties of the perturbation method, it can eventually be used for numerical detection of unsteady bifurcations. Note that the resommation is applied component-wise. This facilitates the parallelisation on multi-processor computers.

Since the resummation method is still almost unknown in the domain of numerical analysis, we give, in the next section, a minimalist reminder of the basic definitions. We then transform the method into a numerical algorithm. In section 3, we analysis the method with the light of some toy examples. In section 4, we show that the resummation method is a good alternative of the adaptative Runge-Kutta method, in the sense that it requires less time steps, for the resolution of ODE's in fluid mechanics. We end up with the numerical resolution of the discretized heat and Burgers equations.

\section{From theory to numerical algorithm}

Maillet's theorem [39] states that if a formal series $\hat{u}$ is a solution of a differential equation with analytical coefficients, then it is a Gevrey series (see definition in 2.1) of some index $m$. Next, the fundamental theorem of Gevrey asymptotics [50] ensures the existence of an holomorphic function which is asymptotic to $\hat{u}$ in some sector, in the Gevrey sense. This function is also a solution of the differential equation. These two theorems guarantee, at least theoretically, the possibility to convert a formal series into an holomorphic function which is a solution of the same problem. The Borel resummation method consists in computing effectively this function, which is its $m$-Borelsum.

In this section, the basic tools needed for the application of the resummation procedure are introduced and some illustrative examples are given. However, only the case $m=1$ is considered in this article. Note that any Gevrey series of index $m \in \mathbb{N}$ can be reduced to a sum of Gevrey series of index one.

For more complete definitions, the reader can refer to Borel and Gevrey's works [9, 30] and to more recent papers on divergent series [8, 50, 48, 29].

\subsection{Basic definitions}

The Gevrey asymptotic theory is a restriction of Poincaré's (standard) asymptotic theory [23]. A Gevrey asymptotic expansion is defined as follows. 
Definition 1. A function $u(t)$ is asymptotic, in Gevrey sense, to a power series $\hat{u}=\sum_{k \geq 0} u_{k} t^{k}$ at the origin in a sector $S$ if, for any compact subsector $T \subset S$, there exists two constants $C$ and $A$ such that

$$
\forall l \geq 1, \forall t \in T, \quad\left|u(t)-\sum_{k=0}^{l-1} u_{k} t^{k}\right|<C A^{l}|t|^{l} l ! .
$$

When it is the case, we write, within this document:

$$
u \stackrel{G}{\sim} \hat{u}
$$

A Gevrey series is a power series which have the same factorial growth as the right-hand side of (4). More precisely,

Definition 2. A formal power series $\hat{u}=\sum_{k \geq 0} u_{k} t^{k}$ is called a Gevrey series if there exists constants $C, A \in \mathbb{R}$ such that

$$
\left|u_{k}\right|<C A^{k} k ! \quad \forall k \geq 1 \text {. }
$$

If $\hat{u}$ is a Gevrey series, then its Borel transform

$$
\mathcal{B} \hat{u}=\sum_{k \geq 0} \frac{u_{k+1}}{k !} \xi^{k} \in \mathbb{C}[[\xi]]
$$

is analytic at the origin.

For an angle $\theta$, we denote $H_{\theta}$ the set of functions which are holomorphic at the vicinity of the origin and in a direction $d_{\theta}=\left[0, \mathrm{e}^{i \theta} \infty[\right.$, decaying exponentially at infinity.

Definition 3. The Laplace transform of a function $P \in H_{\theta}$ in the direction $d_{\theta}$ is

$$
\mathcal{L}_{\theta} P(t)=\int_{0}^{\mathrm{e}^{i \theta} \infty} P(\xi) e^{-\xi / t} d \xi
$$

$\mathcal{L}_{\theta} P$ is defined in a sector bisected by $d_{\theta}$.

Transformation (8) is the formal inverse of the Borel transformation (7) since, formally, $u_{0}+\mathcal{L}_{\theta} \mathcal{B} \hat{u}=\hat{u}$ ( $u_{0}$ has been skipped by the Borel transformation and must be put back). The Borel sum can now be defined. 
THEOREM 4. If, for an angle $\theta, \mathcal{B} \hat{u}$ can be prolonged into a function $P \in H_{\theta}$ then there exists a function $\mathcal{S}_{\theta} \hat{u}$, analytic in a sector of opening $\geq \pi$ bissected by $d_{\theta}$, such that

$$
\mathcal{S}_{\theta} \hat{u} \stackrel{G}{\sim} \hat{u} .
$$

In this case, the formal series $\hat{u}$ is said Borel-summable in the direction $d_{\theta}$.

The Borel sum $\mathcal{S}_{\theta} \hat{u}$ verifies

$$
\mathcal{S}_{\theta} \hat{u}=u_{0}+\mathcal{L}_{\theta} P .
$$

The dependence of the Borel sum on the direction $d_{\theta}$ is related to Stokes phenomenon [33].

In summary, the Borel resummation of a Gevrey series $\hat{u}$ consists in

- computing the Borel transform $\mathcal{B} \hat{u}$,

- extending $\mathcal{B} \hat{u}$ into a function $P \in H_{\theta}$ in Borel space,

- returning to the physical space with the Laplace transformation.

And if $\hat{u}$ is a formal solution of a differential equation then its Borel sum is an analytic solution. These three steps are summarized in Table 1.

$$
\begin{array}{cc}
\hat{u}(t)=\sum_{k \geq 0} u_{k} t^{k} & \mathcal{L}_{\theta} \mathcal{B} \hat{u}(t)=u_{0}+\int_{d_{\theta}} P(\xi) e^{-\xi / t} \mathrm{~d} \xi \\
\text { Borel } & \uparrow \text { Laplace } \\
\mathcal{B} \hat{u}(\xi)=\sum_{k \geq 0} \frac{u_{k+1}}{k !} \xi^{k} \quad \stackrel{\text { Prolongation }}{\longrightarrow} & P(\xi) \in H_{\theta}
\end{array}
$$

Table 1: Resummation steps

Let us illustrate the resummation method with some examples. 


\subsection{Examples}

Consider the Euler equation:

$$
t^{2} \frac{\mathrm{d} u}{\mathrm{~d} t}+u=t
$$

The power series

$$
\hat{u}=\sum_{k \geq 0}(-1)^{k} k ! t^{k+1} .
$$

satisfies formally this equation. Since (11) is divergent, a non-optimized perturbation-method-based algorithm such as ANM would not give any analytical solution. The Borel transform of series (11) is

$$
\sum_{k \geq 0} \frac{u_{k+1}}{k !} \xi^{k}=\sum_{k \geq 0}(-1)^{k} \xi^{k} .
$$

This series can be naturally extended into the analytic function $P: \xi \in$ $\mathbb{C}-\{-1\} \mapsto \frac{1}{1+\xi}$. Hence, the Borel sum of $\hat{u}$ is

$$
\int_{0}^{+\infty} \frac{1}{1+\xi} e^{-\xi / t} \mathrm{~d} \xi
$$

In summary, from the divergent series (11), the resummation method provides the solution (12) of equation (10) which is analytic in the complex half-plane where the real part of $t$ is positive.

In the following example, we consider a problem whose series solution is convergent. We then show that, in this case, the resummation method can be understood as a prolongation method.

Consider the non-linear initial value problem:

$$
\left\{\begin{array}{l}
\frac{\mathrm{d} u}{\mathrm{~d} t}+u^{2}=0 \\
u(0)=1
\end{array}\right.
$$

The formal series solution is:

$$
\hat{u}=\sum_{k \geq 0}(-1)^{k} t^{k}
$$


which is convergent inside the unit disc. The Borel transform of (14) is

$$
\sum_{k \geq 0} \frac{(-1)^{k+1}}{k !} \xi^{k} .
$$

We recognize in (15) the Taylor expansion of the function

$$
\xi \mapsto-e^{-\xi},
$$

that we take as a natural prolongation. The Borel sum is the rational fraction

$$
1-\int_{0}^{+\infty} e^{-\xi} e^{-\xi / t} \mathrm{~d} \xi=\frac{1}{1+t} .
$$

This is the analytic solution of problem (13) in $\mathbb{C}-\{-1\}$. This function extends the series (14) outside its convergence disc. Of course, in this particular case, Padé approximants would give the same solution.

In the next section, the resummation technique is transformed into an algorithm for a numerical use.

\subsection{Algorithm}

On the numerical point of view, the formal power series, obtained with an asymptotic method, is truncated at some order. In the case of partial differential equations, the series coefficients are functions of one ore more variables.

Computing the Borel transform $\mathcal{B} \hat{u}$ presents no particular problem. However, unlike in the linear case, it is not easy to find a differential equation verified by $\mathcal{B} \hat{u}$. Consequently, the singular directions of $\mathcal{B} \hat{u}$ are not known $a$ priori. For this reason, we choose $\theta=0$.

The prolongation in Borel space is materialized via the classical Padé approximants [13]. This choice is motivated by the good ability of this technique to approximate and extend the domain of validity of a series. Another advantage of the Padé approximants (which is not used here) is its ability to detect the singularities of a function through the poles of the rational fraction. Note however that for a high-dimensional problem, typically in fluid mechanics, this method is time-consuming and has to be optimized.

Other prolongation methods are proposed by Thomann in [56] but they are designed for linear equations. 
The Laplace transform is computed using Gauss-Laguerre quadrature [32, 34], which is arbitrarily precise. Six Gauss points are used in the numerical applications. The consistency order can be defined as the truncation order of the initial series, in the sense of (4).

The algorithm is summarized in Table 2.

$$
\begin{array}{cc}
\hat{u}^{l}=\sum_{k=0}^{l} u_{k} t^{k} & \mathcal{L}_{0} \mathcal{B} \hat{u}^{l}(t)=u_{0}+\int_{\mathbb{R}^{+}} P(\xi) e^{-\xi / t} \mathrm{~d} \xi \\
\text { Borel } & \uparrow \begin{array}{l}
\text { Gauss-Laguerre } \\
\mathcal{B} \hat{u}^{l}=\sum_{k=0}^{l-1} \frac{u_{k+1}}{k !} \xi^{k} \stackrel{\text { Padé }}{\longrightarrow}
\end{array} \\
P(\xi)=\frac{A_{0}+A_{1} \xi+\cdots+A_{N_{1}} \xi^{N_{1}}}{1+B_{1} \xi+\cdots+B_{N_{2}} \xi^{N_{2}}}
\end{array}
$$

Table 2: The numerical algorithm

\section{Numerical experiments and continuation procedure}

Consider equation (13), which is not an example on which the maximum profit of the resummation can be made since it does not present any irregularity at the origin, but which has a quadratic non-linearity like the Navier-Stokes equations. The exact solution is

$$
\frac{1}{1+t}
$$

\subsection{Resolution of equation (13)}

In a first test, the solution given by the perturbation method is compared to that provided by the association of the perturbation method and the resummation method. The series solution is computed up to order $l=8$. The result is represented graphically on Figure 1. As observed, the resummation method brings a very significant improvement to the perturbation method. In this case, the range of validity is multiplied by more than 3.5. Note that no 


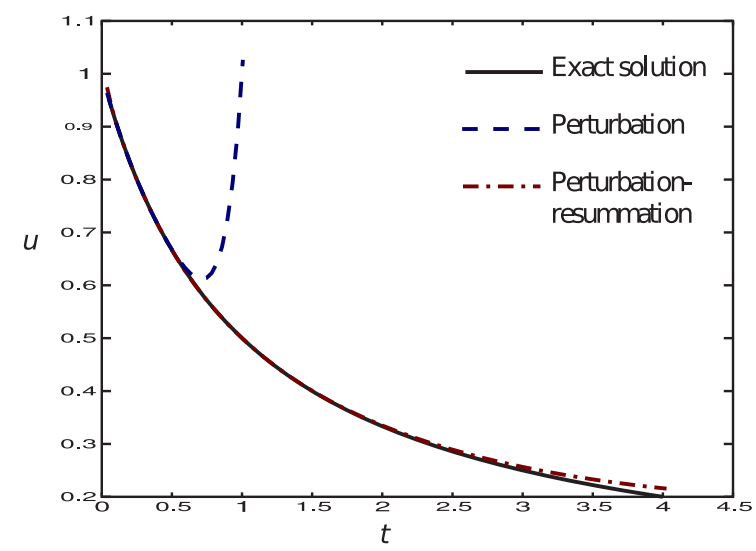

Figure 1: Solutions with and without resummation

optimization operation such as convergence acceleration nor an optimization of the quadrature was performed in the algorithm.

Theoretically, the resummation method should lead to the exact solution until the infinity (see subsection 2.2) but since the initial series was truncated and no optimization was done, numerical effects become non-negligible for large values of $t$.

In order to reach higher values of $t$ with a good precision, a continuation procedure has to be done.

\subsection{Algorithm with continuation procedure}

The accuracy of the approximated solution is appreciated in term of residue, which is defined as

$$
\operatorname{Res}(t)=\frac{\mathrm{d} u}{\mathrm{~d} t}(t)+u^{2}(t) .
$$

The evaluation of (18) necessitates an approximation of $\frac{\mathrm{d} u}{\mathrm{~d} t}$. With the resummation method, this can be done by quadrature method since

$$
\frac{\mathrm{d} u}{\mathrm{~d} t}=\frac{1}{t^{2}} \int_{0}^{\infty} P(\xi) \xi e^{-\xi / t} d \xi
$$

The algorithm, called PERT + RESUM + CONT, which combines the perturbation method, the resummation technique and the continuation procedure can be described as follows. 
a. (PERT) The truncated series

$$
\hat{u}^{l}=\sum_{k=0}^{l} u_{k} t^{k}
$$

is computed using the standard perturbation method, with the initial condition $u_{0}=u\left(t_{0}\right)$. This provides an analytical (actually polynomial) approximation of the exact solution as long as the following relation holds:

$$
\frac{\|\operatorname{Res}(t)\|}{\left\|\hat{u}^{l}(t)\right\|}<\varepsilon
$$

where $\varepsilon$ is a small parameter and $\|\cdot\|$ is the usual Euclidian norm. Let $t_{1}$ be the last computed values of $t$ for which condition (19) holds. $t_{1}$ is equal to zero if the series is divergent.

$\boldsymbol{b}$. (RESUM) Next, the Borel sum $\mathcal{S}_{0} \hat{u}^{l}$ is computed. This provides an analytic approximate solution for $t \in\left[t_{1}, t_{2}\right]$ where $t_{2}$ is the last value of $t$ for which the relative residue is smaller than $\varepsilon$, that is:

$$
\frac{\|\operatorname{Res}(t)\|}{\left\|\mathcal{L}_{0} \mathcal{B}\left(\hat{u}^{l}\right)(t)\right\|}<\varepsilon .
$$

c. (CONT) When $t_{2}$ is reached, a continuation procedure is performed. This mean that the algorithm is run again with, in step $\boldsymbol{a} .,\left(t_{2}, u\left(t_{2}\right)\right)$ as new value of $\left(t_{0}, u_{0}\right)$.

This algorithm is compared to the classical ANM wich is composed of steps $\boldsymbol{a}$. and $\boldsymbol{c}$. $\left(t_{2}=t_{1}\right)$, and which will be refered to as PERT+CONT. These two algorithms are applied to equation (13) in the next section.

\subsection{Equation (13), with continuation procedure}

The computation is carried out up to $t=10$, with $l=8$ and $\varepsilon=10^{-2}$.

Figure 2 shows the approximate solutions. Visually, the exact solution cannot be distinguished from the computed solutions and is not presented. Left is the approximate solution without the resummation procedure. The bold points $(\bullet)$ represent the points where a continuation procedure is needed (the abscissa of these points correspond to the $t_{0}$ 's). On the right side is the 


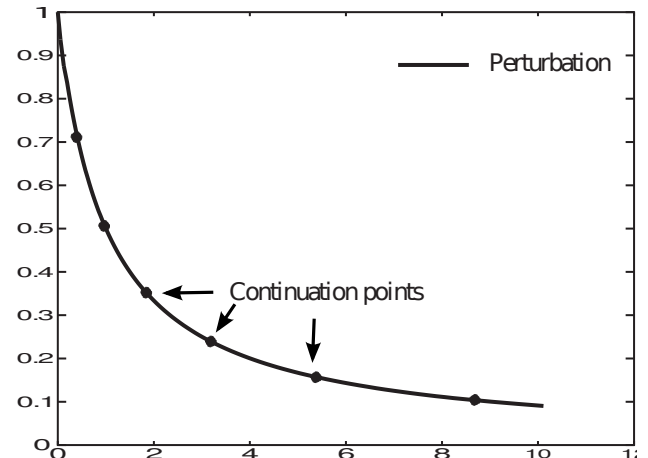

a) $\mathrm{PERT}+\mathrm{CONT}$

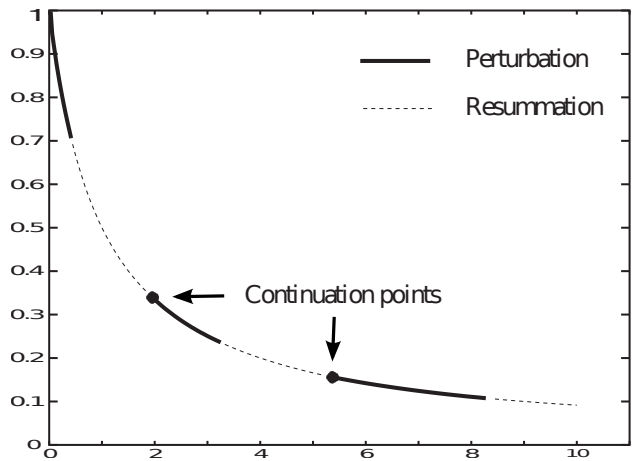

b) $\mathrm{PERT}+\mathrm{RESUM}+\mathrm{CONT}$

Figure 2: Approximated solutions with continuation; a) without resummation, b) with resummation

approximate solution with the resummation procedure. The solid lines correspond to the part of the solution obtained in phase $\boldsymbol{a}$. of the algorithm and the dashes to phase $\boldsymbol{b}$. It can be observed that the resummation procedure prolongs well the solution provided by the perturbation method such that much less continuation steps are needed. Indeed, only two continuation points are required to reach $t=10$ when the resummation method is applied, versus eight when it is not.

The above results correspond to a truncation of the series at order $l=8$. Table 3 compares the number of continuation points with $l=8$ and $l=15$. For $l=15$, a very significant diminution of the number of continuation points can also be observed when the resummation procedure is used.

\begin{tabular}{lcc}
\hline & $\boldsymbol{l}=\mathbf{8}$ & $\boldsymbol{l}=\mathbf{1 5}$ \\
\hline & & \\
PERT + CONT & 6 points & 5 points \\
PERT+RESUM+CONT & 2 points & 1 points \\
\hline
\end{tabular}

Table 3: Number of continuation points for equation (13)

These tests permitted to check that, numerically, the resummation technique improves to the performance of the perturbation method regarding the number of continuation points.

Before considering more realistic problems, let us take a last simple exam- 
ple, whith periodic solution. The analytical solution is know; that permits to analyze the method accuracy and efficiency in terms of computational time, compared to classical time integration methods.

\subsection{A simple periodic problem}

Consider the equations:

$$
\frac{\mathrm{d}}{\mathrm{d} t}\left(\begin{array}{l}
u \\
v
\end{array}\right)=\left(\begin{array}{cc}
0 & -1 \\
1 & 0
\end{array}\right)\left(\begin{array}{l}
u \\
v
\end{array}\right), \quad u(0)=1, v(0)=0 .
$$

$(u, v)$ describes the unit circle. Since the exact solution is known, the maximal relative error of each method can be computed. In this subsection (and only in this one), the quality criterion (20) based on the residue is replaced by another one based on the actual error. The solution is computed within one period $(2 \pi)$.

Table 4 displays the number of steps needed by the resummation method and some classical ones, for given precisions. We observe that the resummation method needs much less steps compared to the usual Euler explicit method and the 2nd and 4th order Runge-Kutta methods. For a precision of $10^{-6}$, the resummation method requires about 10 as less time steps than the 4th order Runge-Kutta method.

\begin{tabular}{lllll} 
Precision & Explicit & RK2 & RK4 & Resum. \\
\hline $10^{-2}$ & 1904 & 65 & 10 & 3 \\
$10^{-3}$ & 19042 & 199 & 17 & 4 \\
$10^{-4}$ & 190400 & 629 & 30 & 5 \\
$10^{-6}$ & $1.910^{7}$ & 6284 & 96 & 9
\end{tabular}

Table 4: Number of steps needed to reach $t=2 \pi$; with explicit Euler method (Explicit), 2nd-order Runge-Kutta method (RK2), 4thorder Runge-Kutta method (RK4) and PERT + RESUM + CONT algorithm (Resum.).

The data on Table 4 are plotted on Figure 3 with logarithmic scales and increasing precision. It can be stated that the resummation method has the smallest slope. Note that the since the explicit method would zoom it out too much, it has not been included in the figure.

The CPU times are reported on figure 4 with logarithmic scales. These values were obtained with an imposed precision of $10^{-6}$ and final times $t_{\max }=$ 1, 2, 10, 100 and 1000 times the period. As shown, the 4-th order Runge-Kutta 


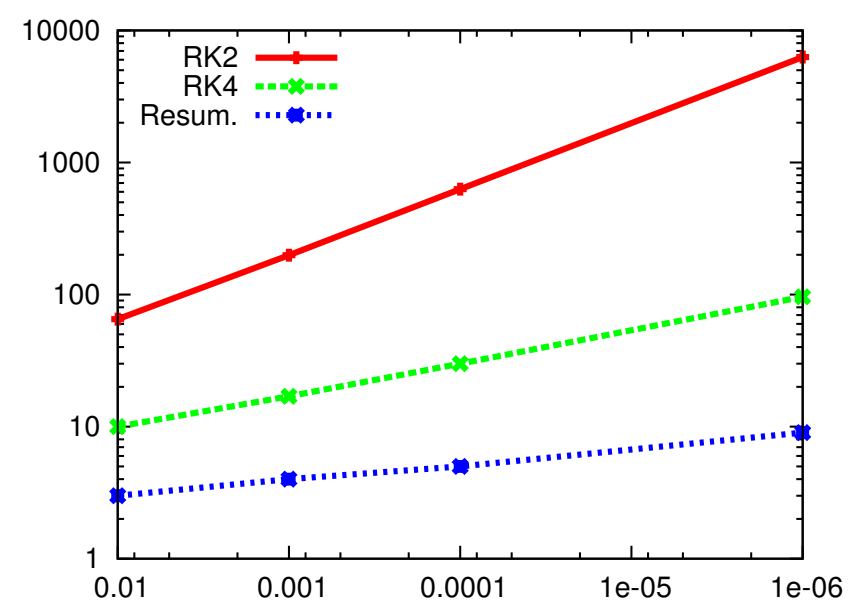

Figure 3: Number of steps needed to reach $t=2 \pi$. See Table 4 for legend.

and resummation methods are comparable in terms of computation time, for small $t_{\max }$. By contrast, for large $t_{\max }$, the gain is clear. For example, to reach 1000 periods with a precision of $10^{-6}$, the resummation method requires $7.210^{-1}$ CPU time, that is 59 times as less than RK4 (4.3 CPU time).

Contrarily to the above examples, the exact solution is not known in real situations and the error of the integration methods is not available. So, in the next cases, comparisons will only be in term of step number. However, in the light of the above example, it is likely that the CPU time increases with the step number.

In the sequel, we consider equations related to fluid mechanics problems, starting with a reduced model of the Navier-Stokes equations for the simulation of realistic fluid flows.

\section{Reduced model of the Navier-Stokes equations}

Consider an incompressible Newtonian fluid of density $\rho$ and kinematic viscosity $\nu$. The motion of this fluid is governed by the Navier-Stokes equations

$$
\left\{\begin{array}{l}
\frac{\partial u}{\partial t}+\operatorname{div}(u \otimes u)=-\frac{1}{\rho} \nabla p+\nu \Delta u \\
\operatorname{div} u=0
\end{array}\right.
$$




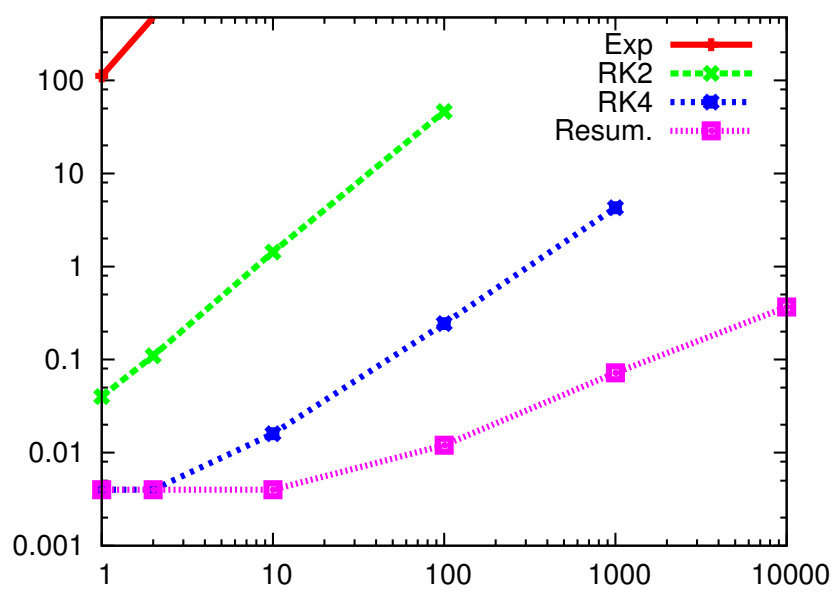

Figure 4: Evolution of CPU time. On the abscissa: $t_{\max } / 2 \pi$.

where $u$ and $p$ are respectively the velocity and pressure fields. It can be shown (see appendix) that a time series decomposition of the spacediscretized solution of (22) leads to a very small radius of convergence, which decreases quadratically with the number of space grid points. This justifies the use of the resummation method as a prolongation procedure. However, in this exploration phase, we will not deal with the whole Navier-Stokes equations but with a reduced model.

Instead, equations (22) are reduced into a dynamical system, using the Proper Orthogonal Decomposition (POD) method which consists in projecting the velocity field $u$ on an optimal basis $\left(\Phi^{i}\right)_{i=1, \ldots, \infty}$, composed of divergent free functions (see [35], [55], [4]):

$$
u(t, x)=\sum_{i=1}^{+\infty} \varrho^{i}(t) \Phi^{i}(x)
$$

The components $\varrho^{i}$ of $u$ will be called the modes. The basis functions are generally determined either numerically or experimentally and depend on the flow configuration.

In practice, the expansion (23) is truncated at an order $m$. This leads to the following reduced model of the Navier-Stokes equations:

$$
\frac{\mathrm{d} \varrho^{i}}{\mathrm{~d} t}+\sum_{j, l=1}^{m} Q_{j l}^{i} \varrho^{j} \varrho^{l}+\sum_{j=1}^{m} L_{j}^{i} \varrho^{j}=F^{i}(t), \quad i=1,2, \ldots m .
$$




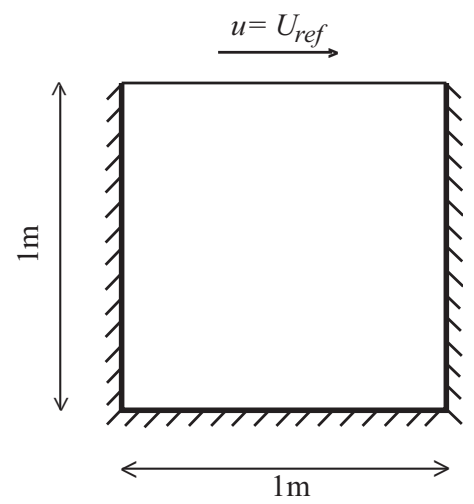

Figure 5: Geometry of the driven cavity

where $Q$ and $L$ are known tensors depending on the basis functions. A property of POD method is that very few modes are enough to capture almost the whole energy of the flow. The term $F^{i}$ contains the pressure and can be vanished, by means of a suitable change of variable, in some cases, such as the followings.

The first test is carried out on an air flow inside a two-dimensional driven cavity, presented on Figure 5. The horizontal lid velocity is $U_{r e f}=0.15 \mathrm{~ms}^{-1}$. The basis functions were computed numerically. They are not presented here but can be found in [1].

Equations (24) are solved using the perturbation-resummation algorithm $(\mathrm{PERT}+\mathrm{RESUM}+\mathrm{CONT})$. Its performance is compared to those of the classical adaptive 5-order Runge-Kutta method in terms of number of time steps. The Runge-Kutta step is chosen such that the estimated value $E$ of the truncation error verifies the following relation:

$$
\frac{E}{\|\varrho(t)\|} \leq \varepsilon
$$

where $\varrho=\left(\varrho^{i}\right)_{i} . \quad \varepsilon$ is the same error parameter used in (19) and (20). Note that the resummation method has an arbitrary order, determined by $l$ (see (4)).

$l$ is set to 10 . The Laplace direction is the positive real axis (it is then assumed that the Padé approximants have no pole on this axis). Four or ten modes $(m=4$ or 10$)$ are computed. The first two modes are presented on Figure 6 up to $t=20 \mathrm{~s}$. A projection on the basis $\left(\Phi^{i}\right)_{i}$ of a numerical solution of the Navier-Stokes equations is used as a reference solution. 

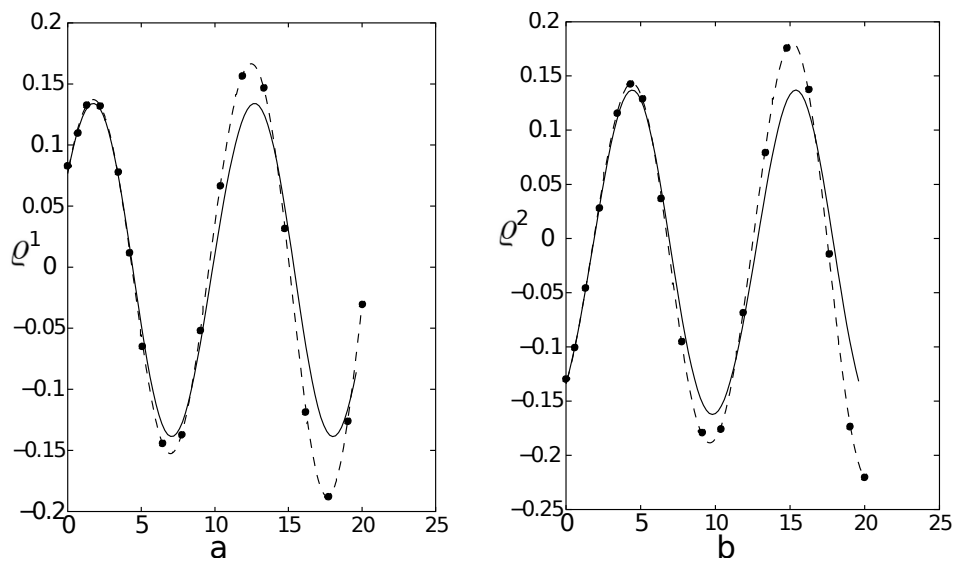

Figure 6: Evolution of $\varrho^{1}$ (a) and $\varrho^{2}$ (b) with $t$

— Reference solution, $\cdots$ Runge-Kutta, - - Perturbation-resummation

It can be observed on Figure 6 that the algorithm PERT + CONT + RESUM gives approximately the same numerical results as the Runge-Kutta method at the points where a Runge-Kutta solution is available. The difference with the reference solution is due to the POD reduction (errors introduced by the computation of the $\Phi^{i}$ s and the truncation of the expansion (23)). Stabilization procedures exist to attenuate the POD modeling errors (see for example $[53,19]$ ) but not used here. The difference with the reference solution is then independent of the performance of our two algorithms.

Figure 7a) compares the number of steps needed to reach 20,50 and 100 seconds for $m=4$ modes $(l=10)$. It shows that the perturbationresummation method requires much less steps than the Runge-Kutta method, about $35 \%$ less to reach 100 seconds.

The gain of the perturbation-resummation method increases with the number of modes. Indeed, as observed on Figure $7 \mathrm{~b}$ ) with $m=10$ modes, the perturbation-resummation method requires about $42 \%$ less continuation steps than the Runge-Kutta method to reach 100 seconds.

From the above results, it can be concluded that the perturbation-resummation method gives the same approximate solution as the Runge-Kutta method, but requires less steps. These results also show that the perturbationresummation method can be used for the simulation of realistic problems. Finally, the gain of the resummation method increases with $m$. It suggests that it is more attractive, compared to the Runge-Kutta method, for the 


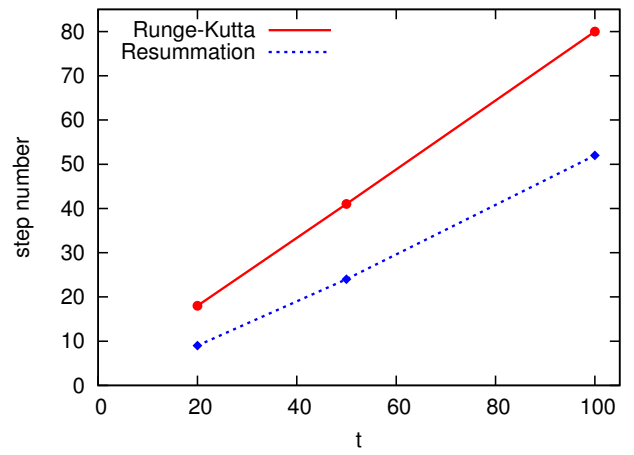

a) with $m=4$ modes

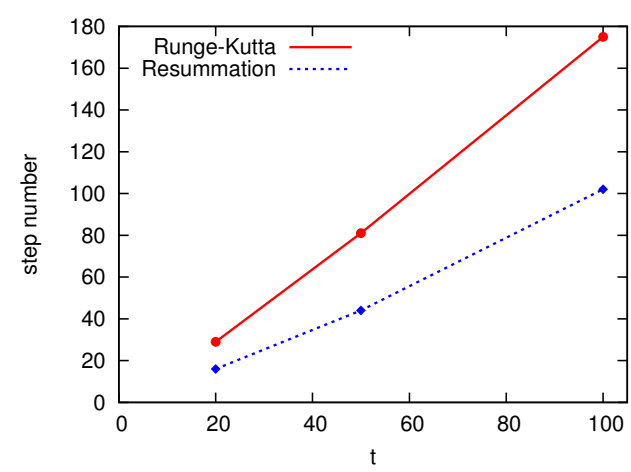

b) with $m=10$ modes

Figure 7: Evolution of continuation step numbers

resolution of high order problems such as the discretized Navier-Stokes equations.

Since the reconstituted flows obtained from the perturbation-resummation method and from the Runge-Kutta method are qualitatively the same, they are not presented in this article but are avalaible in [1].

Another test is carried out on the flow inside the 2D ventilated room presented on Figure 8. This geometry is used in [2] for the study of pollution in buildings. For this geometry, the inlet and outlet heights are $0.31 \mathrm{~m}$. The inlet is at $0.07 \mathrm{~m}$ from the ceiling and the outlet $0.07 \mathrm{~m}$ above the floor. The inlet velocity is $0.443 \mathrm{~m} / \mathrm{s}$.

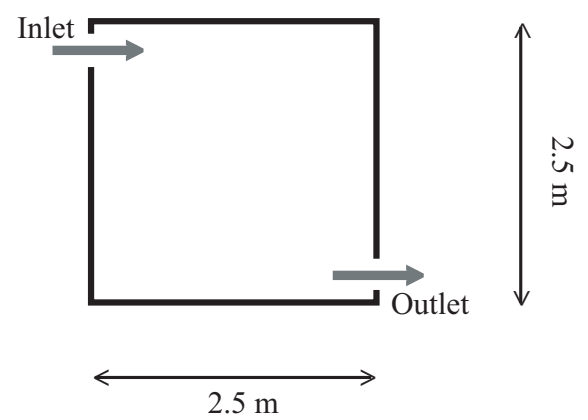

Figure 8: Geometry of the ventilated room

A first simulation is done until the flow is stable. Then, a particle cloud is injected. The details of the simulation can be found in [2] and will not 
be reproduced here. Our interest is the comparison of the perturbationresummation method with the Runge-Kutta method.

The reduction of the equations is particularly interesting in this case because the simulation of particle evolution requires a very small time step if the equations are directly time discretized. The equations are reduced with POD, with $m=4$ modes. Figure 9 presents the number of steps needed by the two methods 20,30 and 100 seconds after the injection of the particles. Also for this flow, the perturbation-resummation method requires less steps than the Runge-Kutta method, about $44 \%$ less to reach 100 seconds. The reconstituted flow can be found in $[2,52]$.

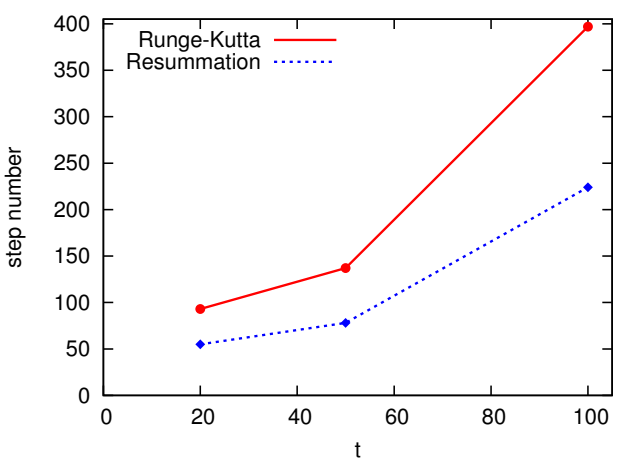

Figure 9: Number of continuation steps for the ventilated room

These numerical tests show that the resummation method may be a good alternative to the Runge-Kutta method, for the resolution of ODE's arising from realistic problems. In the next section, we apply the resummation algorithm to discretized PDE's.

\section{Application to the heat and to Burgers' equations}

Consider the inital-valued heat equation

$$
\left\{\begin{array}{l}
\frac{\partial u}{\partial t}=\nu \frac{\partial^{2} u}{\partial x^{2}} \quad(t, x) \in[0,+\infty[\times \Omega \\
u(t=0, x)=u_{0}(x)
\end{array}\right.
$$


where $\nu$ is the thermal diffusivity coefficient. If $\Omega=\mathbb{R}$, the coefficients of the formal solution

$$
\hat{u}=\sum_{k \geq 0} u_{k}(x) t^{k}
$$

verifies

$$
(k+1) u_{k+1}=\nu \frac{\mathrm{d}^{2} u_{k}}{\mathrm{~d} x^{2}}
$$

that is

$$
u_{k}=\nu^{k} \frac{u_{0}^{(2 k)}}{k !},
$$

The formal solution diverges unless $u_{0}$ is an entire function of exponential order at most 2 (see [36]), that is

$$
\left|u_{0}(z)\right| \leq C \mathrm{e}^{a|z|^{2}}
$$

for some positive constant $C$ and $a$. Series (27) is Borel summable under some conditions on $u_{0}$. For instance, the solution (27) is summable in a sector of opening $>\pi$ if and only if $u_{0}$ is analytic in a double cone and verifies (30) [36], or if $u_{0}$ is a formal series with certain summability properties [5].

In practice, $\Omega$ is a bounded domain and equation (26) is associated to boundary conditions. Herein, Dirichlet boundary conditions of the type

$$
u(t, 0)=u^{0}, u(t, L)=u^{L}
$$

are considered. For the numerical resolution, equations (26) is space-discretized with centered finite difference. This leads to the following equation:

$$
\frac{\mathrm{d} u^{i}}{\mathrm{~d} t}=\frac{u^{i-1}-2 u^{i}+u^{i+1}}{\Delta x^{2}}, \quad i=1, N
$$

where $N$ is the grid size. 32 Gauss points are taken for the Laplace transformation.

Since no matrix inversion is needed with the resummation method, the algorithm PERT + RESUM + CONT can be considered as an explicit method, and is compared to the Euler explicit method in this section. Recall that with the Euler explicit method, the time-step must satisfy the very restrictive stability condition

$$
\Delta t \leq \frac{\Delta x^{2}}{2 \nu}=\Delta t_{E}
$$


For the resummation method, we define a "time step" $\Delta t_{R}$ as the maximal value of $t$ up to which $\mathcal{S}_{0} \hat{u}(t)$ is a good approximation of the solution and at which a continuation procedure must be performed. More precisely,

$$
\Delta t_{R}=t_{2}-t_{0}
$$

where the $t_{i}$ 's are defined in section 3.2. The question is how big $\Delta t_{R}$ is compared to $\Delta t_{E}$. If $\Delta t_{R}>>\Delta t_{E}$, the algorithm PERT + RESUM + CONT is potentially faster than the Euler explicit method.

Two test cases, corresponding respectively to a convergent and a divergent formal solutions are carried out.

\subsection{Heat equation with convergent solution series}

In this subsection, we take $\nu=1, \Omega=[0, \pi]$ and

$$
u(0, x)=\sin x
$$

The exact solution is

$$
u(t, x)=\mathrm{e}^{-t} \sin x
$$

$\Omega$ is discretized into an uniform grid of size $N=16$. The computed solution is presented on Figure 10 for $t \simeq 3 \mathrm{~s}$. It shows a good agreement with the exact solution. The maximum time step with an Euler explicit method would

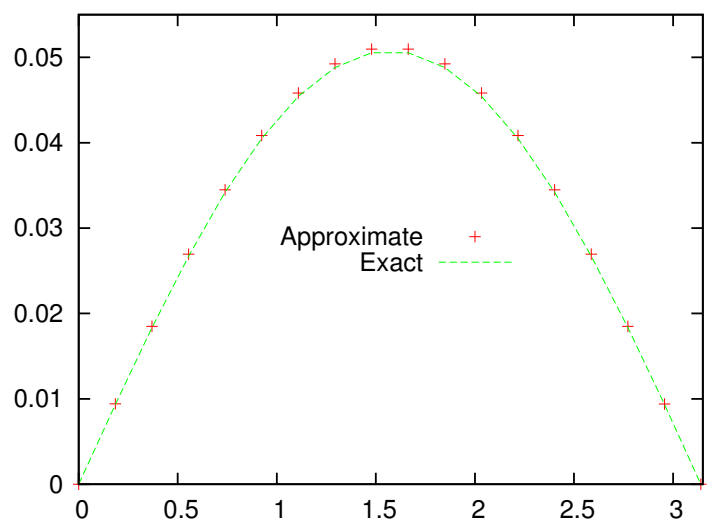

Figure 10: Approximate and exact solutions at $t=2.98 \mathrm{~s}$

be $\Delta t_{E}=1.710^{-3}$. First values of $\Delta t_{R}$, with truncation orders $l=10$ and $l=20$, are shown on Table 5 . It can be observed on it that the first time step 
is much bigger than the next ones. $\Delta t_{R}$ then stabilizes from the second step. Only some values are reported on the table. The last column corresponds to the mean of the 20 first values. It can also be observed that $\Delta t_{R}$ is between 40 and 800 times larger than the time step limit $\Delta t_{E}$ of the Euler explicit method when $l=10$, and between 95 and 900 times larger than $\Delta t_{E}$ when $l=20$.

\begin{tabular}{|c|c|c|c|c|c|}
\hline & step 1 & step 2 & step 3 & step 4 & mean (20) \\
\hline$l=10$ & 1.4 & $8.910^{-2}$ & $9.610^{-2}$ & $9.610^{-2}$ & $1.610^{-1}$ \\
\hline$l=$ & 1.8 & $1.810^{-1}$ & $1.910^{-1}$ & $1.910^{-1}$ & $2.710^{-1}$ \\
\hline
\end{tabular}

Table 5: Time steps with the algorithm PERT + RESUM + CONT

$$
N=16\left(\Delta t_{E} \simeq 210^{-3}\right)
$$

Figure 11, left, presents the mean values of $\Delta t_{R}$, with $N=16$. It can be observed that $\Delta t_{R}$ grows almost linearly with the truncation order $l$. For $l=10$, the right-side of the figure shows that $\Delta_{R}$ decreases quadratically with the grid size, as $\Delta t_{E}$ does. We have:

$$
\Delta t_{R} \simeq 100 \Delta t_{E}
$$
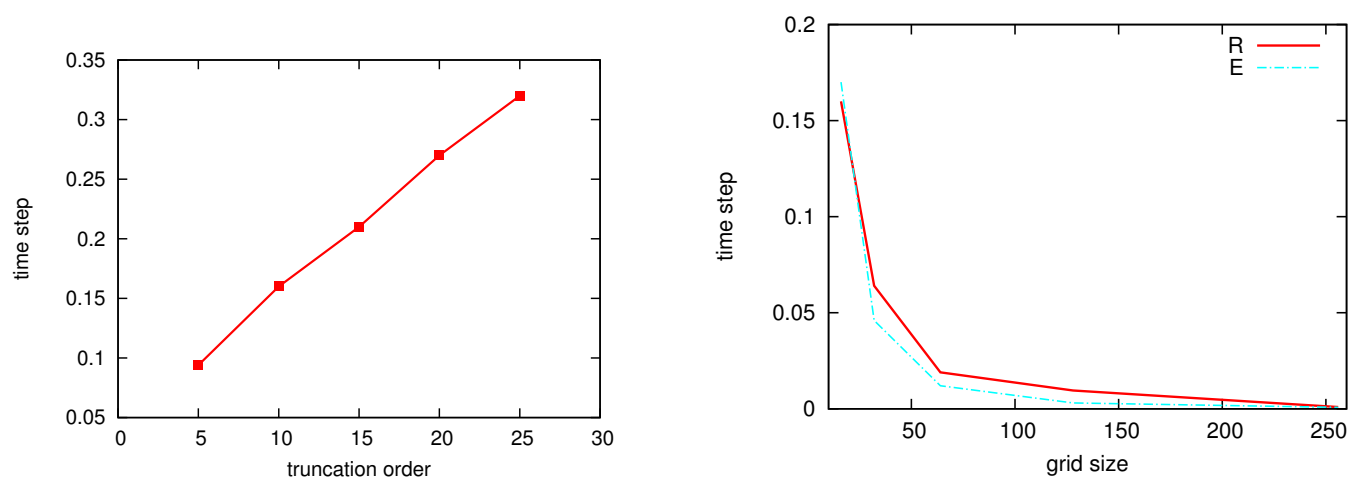

Figure 11: Evolution of mean $\Delta t_{R}$, left: with the truncation order $l$, right: with the grid size $N$ and compared to $100 \Delta t_{E}$ 


\subsection{Heat equation with divergent solution series}

For the last test on the heat equation, we take as initial condition the function proposed by Lutz et al in [36]:

$$
u(t=0, x)=\frac{1}{1-x}
$$

We set the domain to $[0,1 / 2]$ such that function (38) is $C^{\infty}$ inside the domain. The behaviour of the approximate solution for some values of $t$ is sketched on Figure 12. It shows no weird behaviour. Yet the solution series is divergent, as mentionned in introduction.

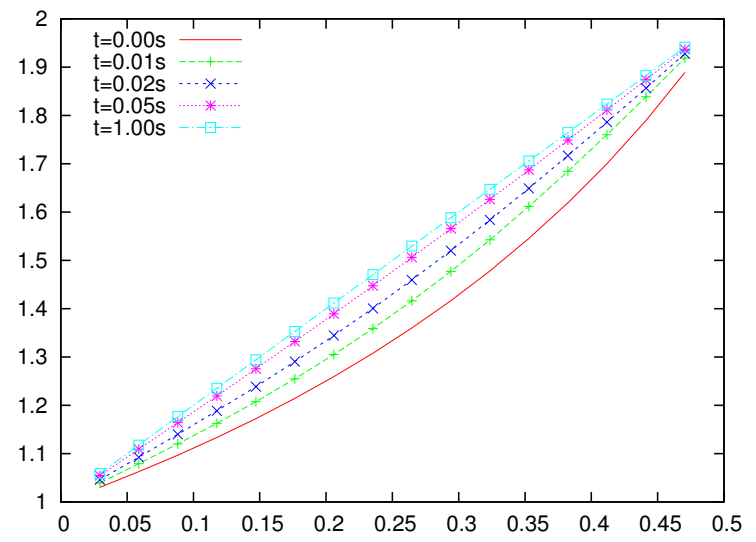

Figure 12: Behaviour of the approximate solution corresponding to initial condition (38)

The goal of this test is to explore numerically the limit of the classical asymptotic method and to confirm the efficiency of the resummation technique for such a problem. The grid size is $N=16$. The time step of the classical asymptotic-method algorithm PERT + CONT is

$$
\Delta t_{A}=t_{1}-t_{0}=t_{2}-t_{0}
$$

where $t_{0}, t_{1}$ and $t_{2}$ are defined in section 3.2.

Figure 13 shows the evolution of the mean (over the first 20 continuation steps) of $\Delta t_{R}$ and $\Delta t_{A}$, when the truncation order is increased. First, we notice that the time step is always larger when the resummation procedure is applied. Next, we observe that, even if it is divergent, the series gives a satisfying approximate solution when only small number of terms are considered $(l<71)$. This is the principle of least term summation. But when the 
truncation order exceeds the limit $l=71$, the classical perturbation method become inefficient. The numerical radius of convergence is zero.

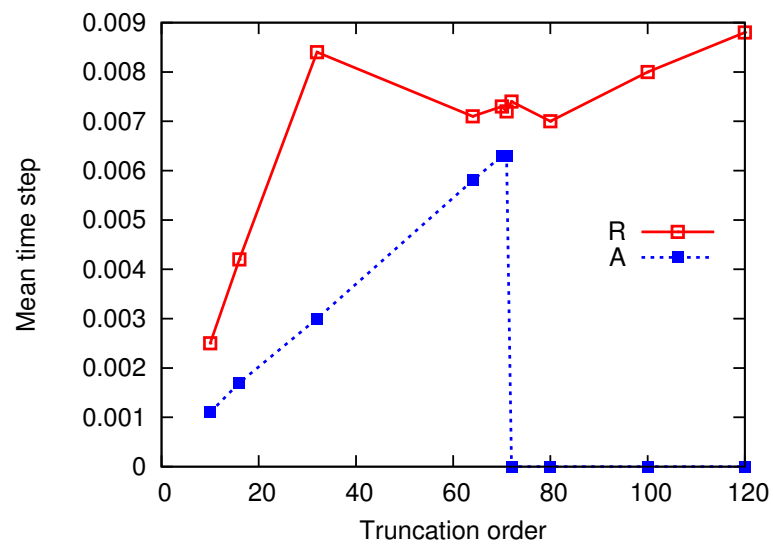

Figure 13: Evolution of the mean time step with the truncation order with algorithms $\mathrm{PERT}+\operatorname{CONT}\left(\Delta t_{A}\right)$ and PERT $+\operatorname{RESUM}+\operatorname{CONT}\left(\Delta t_{R}\right)$

For a bigger problem, for example, when the number of grid point is raised, the critical value of the truncation order decreases. This behaviour is illustrated on Figure 14, where the grid size is increased up to 256. This shows that the resolution of such big problems with the perturbation method without resummation must be limited.

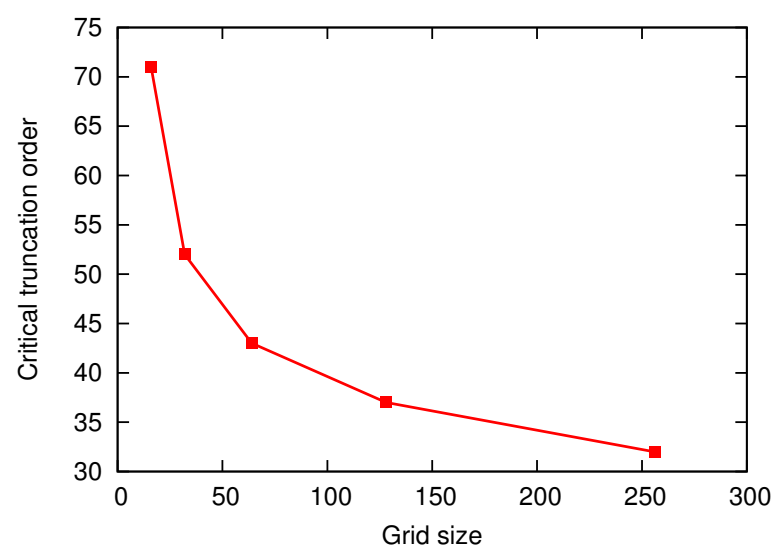

Figure 14: Decay of the truncation order limit (heat equation)

As it can be observed on Figure 13, $\Delta t_{R}$ undergoes a stagnation around 
the critical truncation order but never goes to zero like $\Delta t_{A}$. It even increases again with $l$ beyond the limit $l=71$.

Lastly, we consider Burgers'equation, which is the one-dimensional equivalent of the Navier-Stokes equations.

\subsection{Application to Burgers' equation}

Consider the Burgers'equation with an initial condition:

$$
\left\{\begin{array}{l}
\frac{\partial u}{\partial t}+u \frac{\partial u}{\partial x}=\nu \frac{\partial u}{\partial x^{2}} \\
u(t=0, x)=u_{0}(x)
\end{array}\right.
$$

Without boundary conditions, the coefficients of the formal solution are recursively linked by the relation

$$
(k+1) u_{k+1}=\nu \frac{\mathrm{d}^{2} u_{k}}{\mathrm{~d} x^{2}}-\sum_{p=0}^{k} u_{p} \frac{\mathrm{d} u_{k-p}}{\mathrm{~d} x} .
$$

For $\nu=1$, this relation implies

$$
u_{k}=\frac{1}{k !}\left(u_{0}^{(2 k)}-\sum_{p=0}^{k-1} p ! \sum_{j=0}^{p}\left(u_{j} u_{p-j}\right)^{(2 k-2 p-1)}\right)
$$

Unlike that of the heat equation, the analycity and exponential growth conditions on the initial data $u_{0}$ is not sufficient to ensure the convergence of the formal solution of the Burgers'equation. On the other hand, it is shown in [38] that if $u_{0}$ is analytic, then the formal power series solution is a Gevrey series of order 1 .

For the numerical test, we take the same initial data and boundary conditions as in section 5.2. The formal solution diverges factorially. As seen on Figure 15, the critical truncation order for ANM arises sooner than with the case of the heat equation for small values of $N$. But for large $N$, we have approximately the same limit values.

\section{Conclusion}

We showed that the resummation method may be a good alternative to the Runge-Kutta method for the resolution of ODE's and to the classical 


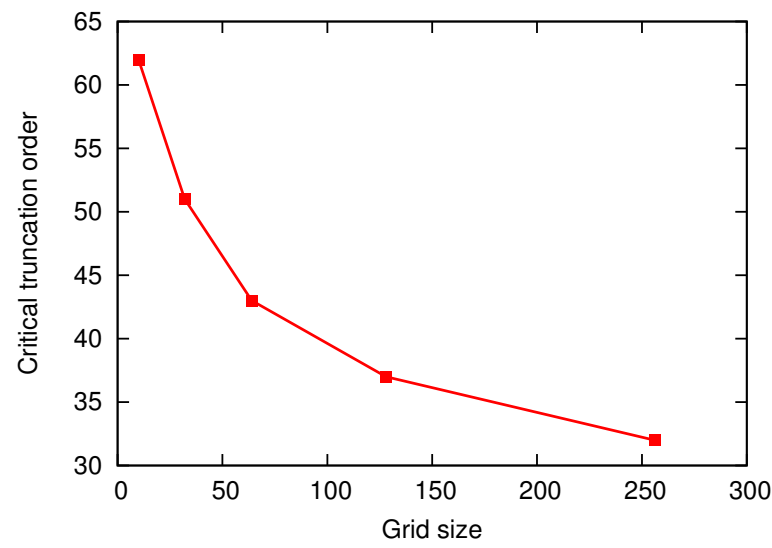

Figure 15: Decay of the truncation order limit (Burgers'equation)

discretization methods (Euler, Runge-Kutta) for the resolution of PDE's in fluid mechanics. For the Navier-Stokes equations, it may become a very interesting tool for the analysis of the behavior of the solution near a blowup time. Note that for these equations, Costin et al. gave Borel-summability conditions of the formal solution and results on the existence of local and global classical solutions [18].

The resummation algorithm seems to be more interesting with large problems. Indeed, the number of steps increases more slowly with the size of the problem, compared to classical methods. But independently of the size, the resummation method appears to be more efficient, both in term of time steps and CPU time, for a long-time problem.

The efficiency can be increased significantly by optimizing the algorithm. For example, the computation of the residue takes an important part of the CPU time in the resolution. Another technique should be used to evaluate the error. Relation (4) can be used to this aim. Note that for the perturbation method and Padé approximants, such methods exist [28]. Other methods for the effective computation of the Borel sum, such as with factorial series [22] or the $(N, \xi)$ approximate summation [45], should also be analyzed. An advantage of the factorial series is that it avoid the use of Padé approximants (which may, as said, not very suitable for fluid mechanics problems) to extend the Borel transform.

Lastly, efforts are still needed to complete the Borel resummation theory for partial differential equations. 


\section{References}

[1] C. Allery. Contribution à l'identification des bifurcations et à l'étude des écoulements fluides par des systèmes dynamiques d'ordre faible (P.O.D.). PhD thesis, 2002.

[2] C. Allery, C. Béghein, and A. Hamdouni. Applying Proper Orthogonal Decomposition to the computation of particle dispersion in a twodimensional ventilated cavity. Communications in Nonlinear Science and Numerical Simulation, 10:907-920, 2005.

[3] C. Allery, S. Guerin, A. Hamdouni, and A. Sakout. Experimental and numerical study of the coanda effect used to reduce self-sustained tones. In FEDSM2002. ASME, 2002.

[4] N. Aubry, P. Holmes, J. Lumley, and E. Stone. The dynamics of coherent structures in the wall region of a turbulent boundary layer. Journal of Fluid Mechanics, 192:115-173, 1988.

[5] W. Balser. Divergent solutions of the heat equation: on an article of Lutz, Miyake and Schäfke. Pacific Journal of Mathematics, 188(1):5363, 1999.

[6] W. Balser. Multisummability of formal power series solutions of partial differential equations with constant coefficients. Journal of Differential Equations, 201(1):63 - 74, 2004.

[7] W. Balser and M. Loday-Richaud. Summability of solutions of the heat equation with inhomogeneous thermal conductivity in two variables. Advances in Dynamical Systems and Applications, 4(2):159-177, 2009.

[8] C. Bender and S. Orszag. Advanced mathematical methods for scientists and engineers : Asymptotic methods and perturbation theory. McGrawHill, 1978.

[9] E. Borel. Mémoire sur les séries divergentes. Annales scientifiques de l'E.N.S. 3ème série, 16:9-131, 1899.

[10] E. Borel. Leçons sur les séries divergentes. J. Gabay edition, 1928. 
[11] F. Boumediene, A. Miloudi, J.-M. Cadou, L. Duigou, and E.H. Boutyour. Nonlinear forced vibration of damped plates by an asymptotic numerical method. Computers and Structures, 87(23-24):1508 - 1515, 2009.

[12] E. Boutyour, H. Zahrouni, M. Potier-Ferry, and M. Boudi. Asymptoticnumerical method for buckling analysis of shell structures with large rotations. Journal of Computational and Applied Mathematics, 168:7785, 2004.

[13] C. Brezinski. Rationnal approximation to formal power serie. Journal of Approximation Theory, (25):295-317, 1979.

[14] J-M. Cadou, M. Potier-Ferry, B. Cochelin, and N. Damil. ANM for stationary Navier-Stokes equations and with Petrov-Galerkin formulation. International Journal for Numerical Methods in Engineering, 50(4):825845, 2001.

[15] J.M. Cadou, M. Potier-Ferry, and B. Cochelin. A numerical method for the computation of bifurcation points in fluid mechanics. European Journal of Mechanics - B/Fluids, 25:234-254, 2006.

[16] B. Candelpergher, J-C. Nosmas, and F. Pham. Approche de la résurgence. Hermann, Paris, 1993.

[17] B. Cochelin. A path-following technique via an asymptotic-numerical method. Computers And Structures, 53(5):1181-1192, 1994.

[18] O. Costin, G. Luo, and S. Tanveer. Divergent expansion, Borel summability and three-dimensional Navier-Stokes equation. Philosophical Transactions of the Royal Society A: Mathematical, Physical and Engineering Sciences, 36(1876):2775-2788, 2008.

[19] M. Couplet, C. Basdevant, and P. Sagaut. Calibrated reduced-order pod-galerkin system for fluid flow modelling. Journal of Computational Physics, 207(1):192-220, 2005.

[20] N. Damil and M. Potier-Ferry. A new method to compute perturbed bifurcations : application to the buckling of imperfect elastic structures. International Journal of Engineering Science, 28(9):943-957, 1990. 
[21] E-M. Daya and M. Potier-Ferry. A numerical method for non-linear eigenvalue problems. Application to vibrations of viscoelastic structures. Computers And Structures, 79:533-541, 2001.

[22] E. Delabaere and J-M. Rasoamanana. Sommation effective d'une somme de borel par séries de factorielles. Annales de l'institut Fourier, 57(2):421-456, 2007.

[23] R. Dingle. Asymptotic expansions: their derivation and interpretation. Academic Press, 1973.

[24] J. Della Dora, C. Di Crescenzo, and E. Tournier. An algorithm to obtain formal solutions of a linear homogeneous differential equation at an irregular singular point. Lecture Notes on Computer Science, 144:273$280,1982$.

[25] J. Della Dora and F. Richard-Jung. About the newton algorithm for non-linear ordinary differential equations. In International Conference on Symbolic and Algebraic Computation, pages 298-304, 1997.

[26] J. Ecalle. Les fonctions résurgentes I: Les algèbres de fonctions résurgentes. Publications Mathématiques d'Orsay, 1981.

[27] J. Ecalle. Les fonctions résurgentes III: L'équation du pont et la classification analytique des objets locaux. Publications Mathématiques d'Orsay, 1985.

[28] A. Elhage-Hussein, M. Potier-Ferry, and N. Damil. A numerical continuation method based on Padé approximants. International Journal of Solids and Structures, 37(46-47):6981 - 7001, 2000.

[29] A. Fruchard and C. Zang. Remarques sur les développement asymptotiques. Annales de la Faculté des Sciences de Toulouse, 8(1):91-114, 1999.

[30] M. Gevrey. Sur la nature analytique des solutions des équations aux dérivées partielles. Premier mémoire. Annales scientifiques de l'E.N.S. 3ème série, 35:129-190, 1918.

[31] Y. Guevel, H. Boutyour, and J.-M. Cadou. Automatic detection and branch switching methods for steady bifurcation in fluid mechanics. Journal of Computational Physics, 230(9):3614-3629, 2011. 
[32] C. Guilpin. Manuel de calcul numérique appliqué. EDP Sciences, 1999.

[33] V. Kowalenko. The Stokes Phenomenon, Borel Summation and MellinBarnes Regularisation. Bentham, 2009.

[34] M. Kzaz. Convergence acceleration of the Gauss-Laguerre quadrature formula. Applied Numerical Mathematics, 29:201-220, 1999.

[35] J. Lumley. The structure of inhomogeneous turbulent flows. In A. Yaglom and V. Tatarski, editors, Atmospheric Turbulence and Radio Wave Propagation, pages 166-178, 1967.

[36] D.A. Lutz, M. Miyake, and R. Schäfke. On the borel summability of divergent solutions of the heat equation. Nagoya Mathematical Journal, 154:1-29, 1999.

[37] G. Lysik. Borel summable solutions of the Burgers equation. Annales Polonici Mathematici, 95(2):187-197, 2009.

[38] G. Lysik and S. Michalik. Formal solutions of semilinear heat equations. Journal of Mathematical Analysis and Applications, 341(1):372 - 385, 2008.

[39] E. Maillet. Sur les séries divergentes et les équations différentielles. Annales scientifiques de l'E.N.S. 3ème série, 20:487-518, 1903.

[40] B. Malgrange. Sur les points singuliers des équations différentielles. L'Enseignement Mathématique, 20:147-176, 1974.

[41] B. Malgrange. Sur le théorème de maillet. Asymptotic Analysis, 2(1):14, 1989.

[42] B. Malgrange and J-P Ramis. Fonctions multisommables. Annales de l'Institut Fourier, 42(1-2):353-368, 1992.

[43] J. Martinet and J.-P. Ramis. Elementary acceleration and multisummability. Annales de l'Institut Henri Poincaré, Physique Théorique, 54:331-401, 1991.

[44] M. Medale and B. Cochelin. A parallel computer implementation of the asymptotic numerical method to study thermal convection instabilities. Journal of Computational Physics, 228(22):8249 - 8262, 2009. 
[45] T. Meurer. Feedforward and feedback tracking control of diffusionconvection-reaction systems using summability methods. VDIFortschrittsberichte, 8(1081), 2005.

[46] S. Michalik. Summability of formal solutions to the n-dimensional inhomogeneous heat equation. Journal of Mathematical Analysis and Applications, 347(1):323 - 332, 2008.

[47] J. Murdock. Perturbation: theory and methods. SIAM, 1999.

[48] F. Pham. Du vieux et du neuf sur les séries divergentes. In Causerie au colloque Emile Borel, Sainte-Afrique, France. PUPE 37, 2000.

[49] J-P. Ramis. Théorème d'indices Gevrey pour les équations différentielles ordinaires. Memoirs of the AMS, (296), 1984.

[50] J-P. Ramis. Séries divergentes et théories asymptotiques. In Journées X-UPS 1991, pages 7-67, 1991.

[51] R. Rand and D. Armbruster. Perturbation Methods, Bifurcation Theory and Computer Algebra. Springer Verlag, 1987.

[52] D. Razafindralandy. Contribution à l'étude mathématique et numérique de la simulation des grandes échelles. Doctoral thesis. Université de La Rochelle, 2005.

[53] D. Rempfer. Kohärente Strukturen und Chaos beim laminar-turbulenten Grenzschichtumschlag. PhD thesis, 1991.

[54] A. Shirai. Maillet type theorem for nonlinear partial differential equations and newton polygons. Journal of the Mathematical Society of Japan, 53(3), 2001.

[55] L. Sirovich. Turbulence and the dynamics of coherent structures, Part I: Coherent strucures, Part II : Symmetries and transformations, Part III : Dynamics and scaling. Quarterly of Applied Machanics, 45(3):561-590, 1987.

[56] J. Thomann. Resommation des séries formelles. Solutions d'équations différentielles linéaires ordinaires du second ordre dans le champ complexe au voisinage de singularités irrégulières. Numerische Mathematik, 58:503-535, 2000. 
[57] E. Tournier. Solutions formelles d'équations différentielles: le logiciel de calcul formel DESIR: étude et réalisation. Phd. Université de Grenoble, 1987.

[58] A. Tri, B. Cochelin, and M. Potier-Ferry. Résolution des équations de Navier-Stokes et détection des bifurcations stationnaires par une méthode asymptotique numérique. Revue Européenne des Eléments Finis, 5(4), 1996.

\section{Appendix A. Time series expansion for the Navier-Stokes equa- tions}

In this appendix, a pertubation-method-based algorithm for the resolution of the discretized Navier-Stokes equations is presented and the convergence radius of the series is estimated. We assume that the domain is bounded.

Consider the Navier-Stokes equation (22), associated to the initial condition

$$
u(0, x)=u_{0}(x)
$$

that we assume analytic within the space domain. After a suitable space discretization, these equations are transformed into a matrix equation:

$$
M \frac{\partial U}{\partial t}+Q(U, U)+L U=0
$$

with

$$
U(t=0)=U_{0}
$$

where $U$ (respectively $U_{0}$ ) are the vectors containing the nodal values of $u$ and $p$ (resp. $u_{0}$ ), $M$ the mass matrix which is equal to the identity matrix if finite difference method is used, $L=\left(L_{j}^{i}\right)_{i, j=1, \ldots, N}$ and $Q$ is a vectorial quadratic operator such that

$$
Q_{i}(V, U)=\sum_{j=1}^{N} \sum_{l=1}^{N} \mathbb{Q}_{j l}^{i} V^{j} U^{l}
$$

$\mathbb{Q}=\left(\mathbb{Q}_{j, l}^{i}\right)_{i, j, l=1, \ldots, N}$ being a three-order tensor and $N$ is the number of grid points. 
The unknown vector $U$ is decomposed into a formal time series as follows:

$$
U(t)=U_{0}+U_{1} t+U_{2} t^{2}+\cdots+U_{k} t^{k}+\cdots
$$

Injecting (A.3) in (A.2) and identifying according to the powers of $t$, one obtains the following cascade of linear equations:

$$
\left\{\begin{array}{cc}
\text { Order } 0: & M U_{1}+Q\left(U_{0}, U_{0}\right)+L U_{0}=0 \\
\text { Order } 1: & 2 M U_{2}+Q\left(U_{0}, U_{1}\right)+Q\left(U_{1}, U_{0}\right)+L U_{1}=0 \\
\vdots & (k+1) M U_{k+1}+\sum_{r=0}^{k} Q\left(U_{r}, U_{k-r}\right)+L U_{k}=0 \\
\text { Order } k: & \vdots
\end{array}\right.
$$

The resolution of these equations provides an approximate solution in the domain of validity of the series (A.3). Since only at most one matrice $(M)$ has to be inverted, the algorithm is explicit. The radius of convergence of (A.3) can be estimated as follows.

For simplicity, we assume that the $M$ is the identity matrix. We denote $U_{k}^{i}$ the $i$ 's component of $U_{k}$ for $k \geq 0$. Define

$$
\mathbf{Q}=\max _{i, j, l=1, \ldots, N}\left|\mathbb{Q}_{j l}^{i}\right|, \quad \mathbf{L}=\max _{i, j=1, \ldots, N}\left|L_{j}^{i}\right|, \quad \mathbf{U}_{\mathbf{0}}=\max _{i=1, \ldots, N}\left|U_{0}^{i}\right|
$$

the respective norms of $\mathbb{Q}, L$ and $U_{0}$. It is straight forward to show that

$$
\left|U_{1}^{i}\right| \leq \mathbf{Q} N^{2} \mathbf{U}_{\mathbf{0}}^{2}+\mathbf{L} N \mathbf{U}_{\mathbf{0}} \leq E
$$

where $E=\left(\mathbf{Q} N^{2} \alpha^{2}+\mathbf{L} N \alpha\right)$ and $\alpha=\max \left(\mathbf{U}_{\mathbf{0}}, 1\right)$. If we assume that

$$
\left|U_{k}^{i}\right| \leq E^{k}, \quad k=1, \ldots, k_{0}
$$

then

$$
\begin{aligned}
\left(k_{0}+1\right)\left|U_{k_{0}+1}^{i}\right| & =\left|\sum_{j=1}^{N} \sum_{l=1}^{N} \mathbb{Q}_{j l}^{i}\left(\sum_{r=0}^{k_{0}} U_{r}^{j} U_{k_{0}-r}^{l}\right)+\sum_{j=1}^{N} L_{j}^{i} U_{k_{0}}^{j}\right| \\
\left(k_{0}+1\right)\left|U_{k_{0}+1}^{i}\right| & \leq \mathbf{Q} \sum_{j=1}^{N} \sum_{l=1}^{N} \sum_{r=0}^{k_{0}} E^{r} E^{k_{0}-r}+\mathbf{L} \sum_{j=1}^{N} E^{k_{0}} \\
& <\mathbf{Q} N^{2}\left(k_{0}+1\right) E^{k_{0}}+\mathbf{L} N E^{k_{0}},
\end{aligned}
$$


that is

$$
\left|U_{k_{0}+1}^{i}\right| \leq\left(\mathbf{Q} N^{2}+\mathbf{L} N \frac{1}{k_{0}+1}\right) E^{k_{0}} \leq E^{k_{0}+1}
$$

This proves by recurrence that

$$
\left|U_{k}^{i}\right| \leq E^{k}, \quad \forall i=1, \ldots, N, k \in \mathbb{N} .
$$

In summary, with a well-behaved initial condition, the formal series of the discretized Navier-Stokes equations decreases quadratically with the grid point, as $E$ does. In the general case, the series is divergent. 